\title{
The Relationship between Glucose Transporter 1 (GLUT1) Expression and the Clinicopathology of Patients with Adenocarcinoma and Squamous Cell Carcinoma Lung Cancer at H. Adam Malik General Hospital Medan
}

\author{
Hutabarat Hotma, Delyuzar, Joko \\ Department of Anatomical Pathology, Faculty of Medicine, Universitas Sumatera \\ Utara, Jalan Universitas No.1, Medan, Indonesia
}

Corresponding author's e-mail: hutabarat.hotma.dr@gmail.com

\begin{abstract}
The largest cause of the incidence and death of cancer patients in the world is due to lung cancer. The biggest energy source for cells is glucose. Glucose transporter 1 is overexpressed in various cancers. Given the importance of GLUT1 in the oncogenesis process, it is necessary to conduct research to determine the prognostic value and subsequent GLUT1 therapy in lung cancer. We collected tissue paraffin blocks formaldehyde from 43 patients with pulmonary Adenocarcinoma (ADC) and Squamous Cell Carcinoma (SqCC) consisting of 34 men and 9 women, used to investigate the immunohistochemical association of GLUT1 expression. Medical records and pathology archives were used as data sources for clinicopathology. The results showed 23 cases (53\%) had GLUT1 expression, while 20 cases $(47 \%)$ were not expressed (negative). Distribution of immunohistochemical expression based on sex was found as many as 21 positive cases $(61.8 \%)$ in male and 2 positive cases $(22,2 \%)$ in female. The mean age of $\mathrm{ADC}$ and SqCC patients was 59,51 years. More than $90 \%$ of people with lung cancer are over 40 years of age. The data from this study also showed a significant relationship between GLUT1 and cancer stage ( $<<0,008$; OR 10,5), Glut 1 with tumor size ( $\mathrm{p}<0,003 ; 18,0)$ and the relationship between GLUT 1 and histopathological type $(p<0,003)$.; OR 9,14). From the results of this study, GLUT1 expression in lung cancer can be an indication of the metabolic and vascular requirements of tumors with clinical implications for patient survival and lung cancer follow-up therapy plans
\end{abstract}

Keyword : Glucose Transporter 1 (GLUT1), pulmonary Adenocarcinoma, pulmonary Squamous Cell carcinoma

\section{Introduction.}

It is estimated that there will be an increase in cancer problems worldwide to 18,1 million new cases with 9,6 million deaths in 2018. Data shows that 2,1 million new cases and 1,8 million deaths are due to lung cancer as the main cause of cancer in 2018.[1] This is due to the poor prognosis for the disease worldwide.[2] The type of cancer most often diagnosed in men is lung cancer, accounting for $14,5 \%$ of all cases in men and $8,4 \%$ in women, and lung cancer is the leading cause of cancer death in men $(22,0 \%)$.[1] The use of glucose in cancer cells shows a higher metabolic rate compared to normal cells to support their proliferation ability.[3] The increased need for glycolysis in cells as well as the use of glucose to produce energy in cancer is known as the "Warburg effect". There are 14 family members of GLUT and the most frequent cause of cancer in humans and is responsible for meeting high glucose 
requirements is the task of GLUT1.[4] The oncogenesis process can show excess GLUT 1 in various cancers, as a result of the high demand for glucose in the cytoplasm of tumor cells. [5] The process of resetting cancer cell metabolism leads to high proliferation of cancer cells.[5] High GLUT1 expression as an indication of hypoxia and glucose metabolism. So that researchers feel the need to conduct research on GLUT1 to see its prognostic value in lung cancer types $\mathrm{ADC}$ and $\mathrm{SqCC}$.

\section{Materials and Methods}

We studied 43 sample of pulmonary Adenocarcinoma and Squamous Cell Carcinoma at the Department of Anatomic Pathology, Universitas Sumatera Utara/H. Adam Malik General Hospital Medan, using the cross sectional approach. We collected clinical data including age, gender, smoking status, tumor stage, tumor size, tumor location and histopathology type. The histopathology type was evaluated by three investigators through microscopic examination of the slides with hematoxylin and eosin staining.

Immunohistochemical staining was performed using GLUT1 (Glucose Transporter 1), rabbit polyclonal antibody, Bioassay Technology Laboratory. Positive control is endometrial tissue (Adenocarcinoma endometrium). The GLUT1 expression was evaluated by three researchers by summing the results of the area score with the intensity score. So that the immunoreactive GLUT1 score is obtained. The broad score assessment (score 0-3) and intensity score assessment (score $0-3$ ), with a total score of 6 , where the score $0-4$ is called a negative GLUT1 expression and a score 5-6 is called a positive GLUT1 expression.[6] GLUT1 expression was found to be positive in the cytoplasm and cytoplasmic membrane.

\section{Results}

Table 1. Data on clinicopathology distribution of samples of lung cancer patients

\begin{tabular}{|c|c|c|}
\hline Clinicopathological & Number of case (n) & Percentage $(\%)$ \\
\hline \multicolumn{3}{|l|}{ Gender } \\
\hline Male & 34 & 79 \\
\hline Female & 9 & 21 \\
\hline \multicolumn{3}{|l|}{ Age (years) } \\
\hline$<65$ years & 30 & 70 \\
\hline$\geq 65$ years & 13 & 30 \\
\hline \multicolumn{3}{|l|}{ Smoking status } \\
\hline Do not smoke & 6 & 14 \\
\hline Smoke & 37 & 86 \\
\hline Light & 2 & 5,4 \\
\hline Moderate & 9 & 24,3 \\
\hline Heavy & 26 & 70,3 \\
\hline \multicolumn{3}{|l|}{ Tumor stage } \\
\hline Stage I & 6 & 14 \\
\hline Stage II & 6 & 14 \\
\hline Stage III & 11 & 25,5 \\
\hline
\end{tabular}


Tumor size

Stage IV 20

$<3 \mathrm{~cm}$

$\geq 3 \mathrm{~cm} \quad 33$

Location of the tumor

Central 21

Perifer 22

Histopathology type

Adenocarcinoma

Squamous cell carcinoma
20

46,5

23

77

49

51

53

47

This study found 23 cases (53\%) of patients who expressed positive with GLUT1 and 20 cases (47\%) who did not express GLUT1 (Table 2).

Table 2.Data on the distribution of GLUT1 immunohistochemical expression

\begin{tabular}{lcc}
\hline Expression GLUT1 & Number of cases(n) & Percentage (\%) \\
\hline Negative (-) & 20 & 47 \\
Positive (+) & 23 & 53 \\
\hline Total & 43 & 100 \\
\hline
\end{tabular}

Based on the results of this study, the distribution of GLUT1 immunohistochemical expression was obtained by 21 positive samples $(61,8 \%)$ in male, while for female, 2 samples $(22,2 \%)$ and 7 negative samples $(77,8 \%)$ were obtained. For the distribution of GLUT1 expression based on age, it was obtained data that age $<65$ years were positive as many as 16 samples $(53,3 \%)$ and negative 14 samples $(46,7 \%)$ while for those aged $\geqslant 65$ years who were positive totaled 7 samples $(53,8 \%)$ and 6 samples expressed negative $(46,2 \%)$. Smoking status shows the same data for positive and negative expressions on GLUT1, namely 3 samples (50\%) of all existing samples. For patients who smoke, it was found that 20 samples $(54,1 \%)$ expressed positive with GLUT1 and 17 samples $(45,9 \%)$ were not expressed (negative). From the data on the distribution of GLUT1 immunohistochemical expression based on tumor stage I and II, there were 2 samples $(16,7 \%)$ that expressed positive and 10 samples $(83,3 \%)$ were not expressed (negative), while for stages III and IV there were 21 samples. $(67,7 \%)$ who expressed positive and 10 samples $(32,3 \%)$ were negative. For the location of the tumor in the center there were 12 samples $(57,1 \%)$ and 9 samples $(42,9 \%)$ that were not expressed. Researchers then performed statistical tests, and obtained results that showed relationship between GLUT1 and tumor stage $(\mathbf{p}<\mathbf{0 , 0 0 8}$; OR: 10,5), tumor size $(\mathbf{p}<\mathbf{0 , 0 0 3}$; OR: 18,0) and histopathology type (p<0,003; OR: 9,14). 
Table 3. Data on the distribution of GLUT1 immunohistochemical expression based on the clinicopathology of patients.

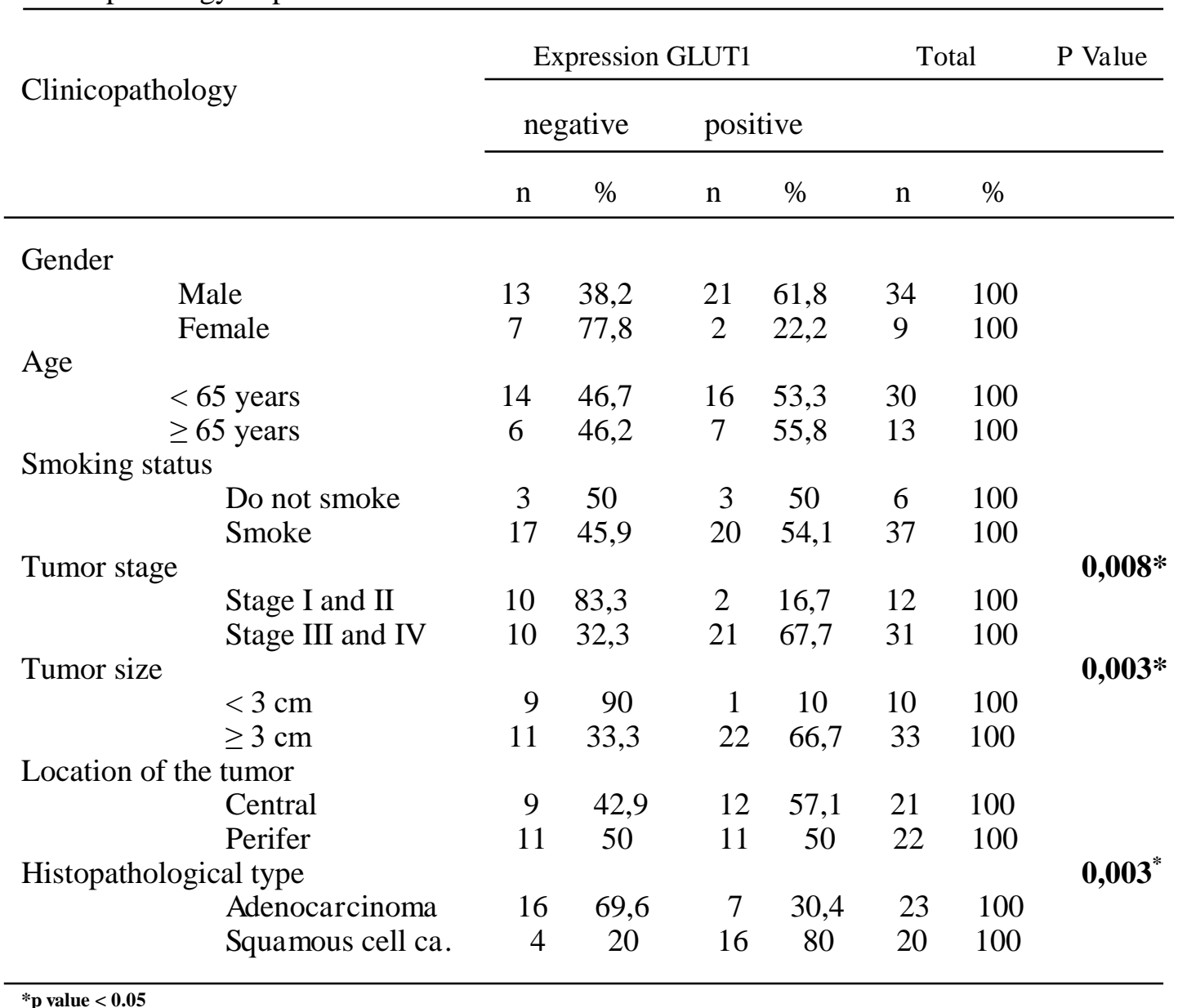
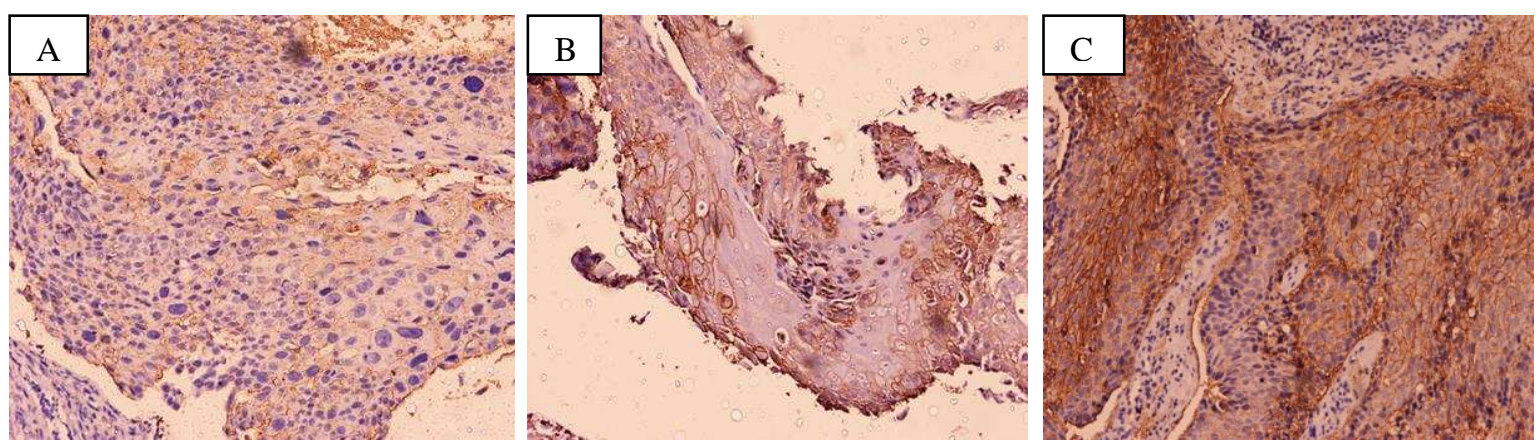

Figure 1. A.GLUT1 expression in SqCC (+1), B. GLUT1 expression in SqCC (+2), C. GLUT1 expression in $\mathrm{SqCC}(+3)$ 

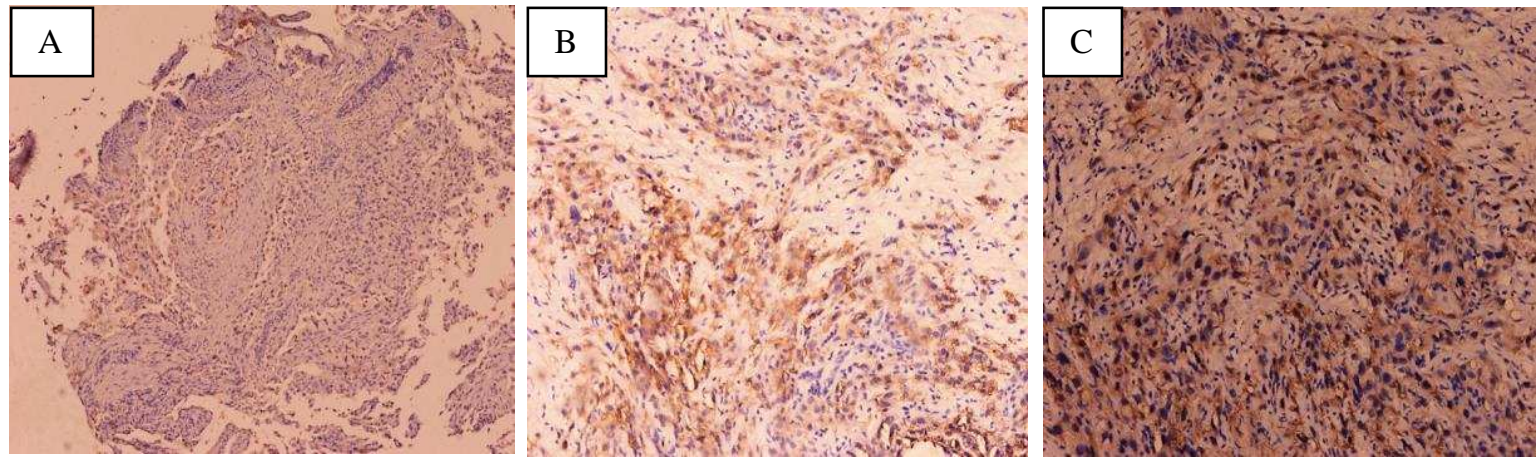

Figure 2. A.GLUT1 expression in ADC (+1), B. GLUT1 expression in ADC (+2), C. GLUT1 expression in $\mathrm{ADC}(+3)$

\section{Discussion}

The nutritional requirement of the tumor grows according to its volume, but its ability to absorb materials by diffusion from the surrounding tissue corresponds to the surface area of the tumor. Therefore the tumor grows to its maximum size, as a result, the tumor is deficient in nutrients which will inhibit tumor proliferation until the tumor is in a silent state.[7] Squamous cell carcinoma has a smaller number of blood vessels, indicating poor blood perfusion in the tumor, leading to hypoxia and increased glucose requirements for metabolism.[8]

The features of SqCC often show necrosis caused by the presence of hypoxia and a higher rate of glycolysis, while the level of oxidative phosphorylation is higher in ADC.[8] Anaerobic (hypoxic) glycolytic conditions were found in SqCC which consisted of poor vascularization, extensive necrosis, and high lactate, as well as increased expression of GLUT1, whereas aerobic conditions were found in ADC which had good vascularity, and glycolytic metabolism.[9]

Studies have shown that SqCC exhibits higher expression in GLUT1 compared to ADC, as a result of the presence of ischemia, hypoxia or both.[8] Adenocarcinoma and SqCC showed different patterns of GLUT1 expression in location and frequency in tumors. Tumor cells are highly dependent on glycolysis with lactate production rather than mitochondrial oxidation, even in the presence of oxygen (Warburg effect) or 'aerobic glycolysis'.[10] To support high levels of glycolysis, tumor cells require increased glucose transport into the cells. This is facilitated by upregulation of glucose transport (GLUTs).[10]

These results indicate that pulmonary $\mathrm{ADC}$ and pulmonary $\mathrm{SqCC}$ exhibit different metabolisms.[11] In Adenocarcinoma, cytoplasmic staining patterns and diffusion are observed along with membrane staining. In contrast, Squamous Cell Carcinoma expresses GLUT1 exclusively within the membrane.[4] This study shows that GLUT1 in SqCC is localized on the plasma membrane and plays a role in increasing glucose intake.[4] GLUT 1 
expression is lower in ADC because ADC is independent of glucose metabolism.[4] Glucose uptake and GLUT1-mediated metabolism in SqCC can be utilized for the development of targeted therapeutic strategies for $\mathrm{SqCC}$.

\section{Conclusion}

After conducted this research, we highlighted several points in the following

1. From the results of the study, it was found that lung cancer patients for the male gender were more dominant than for female.

2. Sufferers who smoke more than those who don't.

3. There is a significant relationship between GLUT1 with tumor stage, tumor size and histopathological type.

\section{Acknowledgement}

We would like to thank all those who helped in completing this research.

\section{References}

[1] International Agency for Research on Cancer, Latest global cancer data : cancer burden rises to 18,1 million new cases and 9,6 million cancer deaths in 2018, 12 September 2018

[2] Bray F, Ferlay J, Soerjomataram I. Global Cancer Statistics 2018 : GLOBOCAN Estimates of Incidence and Mortality Worldwide for 36 Cancers in 185 Countries. 2018;394-424.

[3] Tan Z, Yang C, Zhang X, Zheng P, Shen W. Expression of glucose transporter 1 and prognosis in non-small cell lung cancer: a pooled analysis of 1665 patients. 2017;8(37):60954-61.

[4] Goodwin J, Neugent ML, Lee SY, Choe JH, Choi H, Enkins DMRJ, et al. The distinct metabolic phenotype of lung squamous cell carcinoma defines selective vulnerability to glycolytic inhibition. Nat Commun [Internet]. 2017;8(May 2016):1-16. Available from: http://dx.doi.org/10.1038/ncomms 15503 .

[5] Zhang B, Xie Z, Li B. The clinicopathologic impacts and prognostic significance of GLUT1 expression in patients with lung cancer: a meta-analysis, Department of Thoracic Oncology, First Affiliated Hospital of Xi'an Medical College, Xi'an 710032, Shaanxi Province, P.R. China 2 Burns Center PLA, Department Cutaneous Surgery, Xijing Hospital, Air Force Military Medical University, Xi'an 710032, Shaanxi Province, P.R. China. 2018.

[6] Kato $\mathrm{Y}$, Nishihara H, Mohri H, Kanno H, Kobayashi H, Kimura T, et al. Clinicopathological evaluation of cyclooxygenase-2 expression in meningioma: Immunohistochemical analysis of 76 cases of low and high-grade meningioma. Brain Tumor Pathol. 2014;31(1):23-30. 
[7] Plank, MJ and Sleeman BD. Tumor-Induced Angiogenesis: A Review Journal of Theoretical Medicine, Vol.5,pp.137-53. 2003.

[8] Schuurbiers OCJ, Meijer TWH, Kaanders JHAM, Looijen-Salamon MG, De Geus-Oei LF, Van Der Drift MA, et al. Glucose metabolism in NSCLC is histology-specific and diverges the prognostic potential of 18FDG-PET for adenocarcinoma and squamous cell carcinoma. J Thorac Oncol. 2014;9(10):1485-93.

[9] Zhang J, Chen L, Chen Y, Wang W, Cheng L, Zhou X, et al. Tumor vascularity and glucose metabolism correlated in adenocarcinoma, but not in squamous cell carcinoma of the lung. PLoS One. 2014;9(3):1-10.

[10] Meijer TWH, Schuurbiers OCJ, Kaanders JHAM, Looijen-Salamon MG, de Geus-Oei LF, Verhagen AFTM, et al. Differences in metabolism between adeno- and squamous cell non-small cell lung carcinomas: Spatial distribution and prognostic value of GLUT1 and MCT4. Lung Cancer [Internet]. 2012;76(3):316-23. Available from:http://dx.doi.org/10.1016/j.lungcan.2011.11.006

[11] Zhang B, Xie Z, Li B. The clinicopathologic impacts and prognostic significance of GLUT1 expression in patients with lung cancer: A meta-analysis. Gene 2019. 